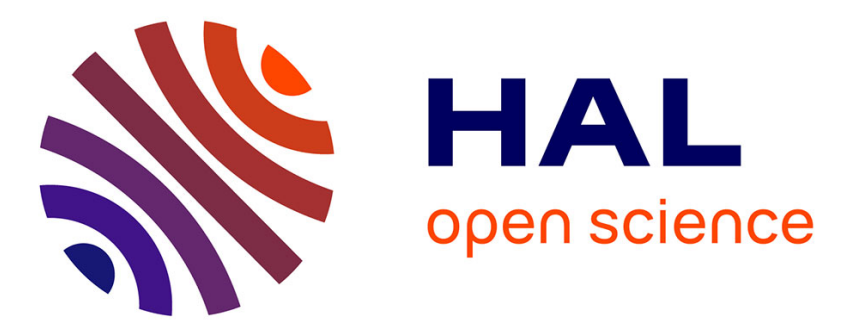

\title{
Contactless data transfer for autonomous underwater vehicle docking station
}

Pierre-Jean Bouvet, Philippe Forjonel, Béatrice Tomasi, Yves Auffret, Yassine Amirat, Gilles Feld, Maëlle Deniel, Pauline Simon

\section{> To cite this version:}

Pierre-Jean Bouvet, Philippe Forjonel, Béatrice Tomasi, Yves Auffret, Yassine Amirat, et al.. Contactless data transfer for autonomous underwater vehicle docking station. OCEANS 2021, Sep 2021, San Diego, CA, United States. hal-03454717

\section{HAL Id: hal-03454717 https://hal.science/hal-03454717}

Submitted on 29 Nov 2021

HAL is a multi-disciplinary open access archive for the deposit and dissemination of scientific research documents, whether they are published or not. The documents may come from teaching and research institutions in France or abroad, or from public or private research centers.
L'archive ouverte pluridisciplinaire HAL, est destinée au dépôt et à la diffusion de documents scientifiques de niveau recherche, publiés ou non, émanant des établissements d'enseignement et de recherche français ou étrangers, des laboratoires publics ou privés. 


\section{Contactless data transfer for autonomous underwater vehicle docking station}

\author{
Bouvet Pierre-Jean \\ $L @ b I S E N$ \\ ISEN Yncréa Ouest \\ Brest, France
}

pierre-jean.bouvet@isen-ouest.yncrea.fr

\author{
Auffret Yves \\ L@bISEN \\ ISEN Yncréa Ouest \\ Brest, France \\ yves.auffret@isen-ouest.yncrea.fr
}

\author{
Deniel Maëlle \\ ISEN Yncréa Ouest \\ Brest, France \\ maella.deniel@isen-ouest.yncrea.fr
}

Forjonel Philippe

L@bISEN

ISEN Yncréa Ouest

Brest, France

philippe.forjonel@isen-ouest.yncrea.fr

\author{
Amirat Yassine \\ L@bISEN \\ ISEN Yncréa Ouest \\ Brest, France \\ yassine.amirat@isen-ouest.yncrea.fr
}

\author{
Tomasi Beatrice \\ L@bISEN \\ ISEN Yncréa Ouest \\ Brest, France
}

beatrice.tomasi@isen-ouest.yncrea.fr

\author{
Simon Pauline \\ ISEN Yncréa Ouest \\ Brest, France \\ pauline.simon@isen-ouest.yncrea.fr
}

\begin{abstract}
Due to the water's characteristics, especially salt water, electromagnetic fields are quickly dissipated and absorbed in underwater environment. Thus, to transfer data in this medium, acoustic signals are mostly used due to their capability to transmit data over a long range. However, at very short range, electromagnetic signals enable high rate wireless data transfer in the underwater medium. The objective of this paper is to investigate underwater contactless data transfer technologies between an Autonomous Underwater Vehicle (AUV) and its docking station. On one hand, we consider $5 \mathrm{GHz}$ Wi-Fi based transmission prototype and provide experimental end-to-end data performance for several transmission range, rotation angles, non-perfect alignment and transmit power. On the other hand we investigate the achievable rate of an experimental Magnetic Induction (MI) prototype performing contactless power and data transfer.
\end{abstract}

Index Terms-Contactless, underwater communication, Wi-Fi, induction

\section{INTRODUCTION}

Nowadays Autonomous Underwater Vehicle (AUV) are extensively used for undersea exploration and monitoring with many applications in the military, scientific or commercial fields. Within the European project called Integrated Technologies Longterm Deployment of Robotic Underwater Platforms (INTENDU), partners from France, Portugal, and Norway aim to develop technologies that improve the current capabilities of AUVs by enabling them to be deployed for extended periods of time. A specific application targeted by the project is the long-term monitoring of fish-farming infrastructures through AUVs.

In the framework of INTENDU project, an innovative docking power hub based on Inductive Contactless Power
Transfer (ICPT) has been developed in order to charge AUVs under water, as described in [1]. In the framework of a docking station providing both power transfer and data connectivity to resident AUVs, the next step lies in data transfer between AUVs and docking station (and reversely) by using contactless connectors. The need for underwater wireless communications with underwater vehicles has led to extensive research in the last decades by using either acoustic, optical or electromagnetic signals [2].

Underwater acoustic communication systems achieve long range (up to hundred kilometers) but exhibit poor communication performance especially in shallow water and are constrained to limited bandwidth leading in practice to low data rate [3]. Optical systems allow high bandwidth at closer range (up to $100 \mathrm{~m}$ ) but are very sensitive to turbidity and require relative good line-of-sight alignment that limits operational scenario especially in coastal areas. On the other hand, underwater Radio Frequency (RF) communication systems have been investigated by the research community as an interesting alternative to optical and acoustic underwater links since they provide the advantage of not being affected by turbidity, operate in non-line of sight, and provide high data rate at very close range [4], [5].

The objective of this paper is to experimentally study technologies for contactless data transfer with electromagnetic waves in the underwater medium by considering on one hand IEEE 802.11ac standard on Super High Frequency (SHF) bandwidth owing to Commercial off-the-shelf (COTS) RF components, and on the other hand Mutual Induction (MI) communication capabilities offered by the previously 
developed ICPT system in the framework of AUV docking charging hub [1]. The remainder of this paper is organized as follows. Section II summarizes the theoretical attenuation of $5 \mathrm{GHz}$ Wi-Fi signal underwater and describes the associated transmission prototype. In section III we introduce the ICPT system and the achievable rate of the MI communication prototype. Section IV provides experimental results of both Wi-Fi and MI communications. Finally conclusions are drawn in Section V.

\section{IEEE 802.11 AC 5GHz WIFI UNDERWATER COMMUNICATIONS}

A contactless wireless data transfer prototype based on the 802.11ac standard was developped and tested in air, fresh and salt water.

\section{A. Theoretical attenuation of a $5.2 \mathrm{GHz}$ Wi-Fi signal}

As pinpointed in the literature [6], a Wi-Fi communication system can ensure large data payload transfers from an AUV to its docking station underwater but over very short ranges. In fact, attenuation in fresh (salt) water of the Wi-Fi $5.2 \mathrm{GHz}$ signal is demonstrated to be around $1000 \mathrm{~dB} / \mathrm{m}(1800 \mathrm{~dB} / \mathrm{m})$, as shown in [7]. This large attenuation makes COTS RF components suitable for transmitting data over very short ranges. We used a ubiquiti Rocket 5 AC-Lite modem transmitting a 30 $\mathrm{dBm}$ maximum signal level and receiving at least $-96 \mathrm{dBm}$ power signal. In this case, we measured a power dynamic of $126 \mathrm{dBm}$ absorbed in less than $13 \mathrm{~cm}$ in fresh water.

\section{B. Methodology and measurement system}

The prototype, shown in Fig. 1, is composed of two Ubiquiti airMAX Rocket AC Lite $5.2 \mathrm{GHz}$ modems with two selfmade quarter-wave antenna for each equipment. One modem is integrated in the docking station side, PoE-powered, the other modem is at the AUV side, battery-powered connected to a ubiquiti's UVC G4 Pro camera. The maximum data rate in the air for this modem in 256-QAM is $400 \mathrm{Mbps}$.

The main goal of this experiment is to characterize the data rate from the AUV to the docking station over a range of up to $13 \mathrm{~cm}$. During the experiment, all the data is collected in influxDB database thanks to Simple Network Management Protocol (SNMP) agent in the docking station modem and then analyzed in a Grafana interface. The tube containing the modem at the AUV side can slide in the enclosure clamp: a ruler underneath indicates the distance between the tubes. The rotation can be observed by markers on the system, and to perform misalignment, the enclosure clamp can be moved by centimeter steps towards left or right directions.

The prototype is immersed in a water tank so that the modems are separated by more than $13 \mathrm{~cm}$ from a surface or the air as we can see in Fig. 1. The water for the experiment is either fresh water or salt water $(10 \mathrm{~kg}$ of salt in $300 \mathrm{l}$ of fresh water). The data rate tests consist in making the AUV modem transmit data at its maximum rate during 99 seconds and in monitoring the performance of this communication at the receiving modem placed in the docking station..
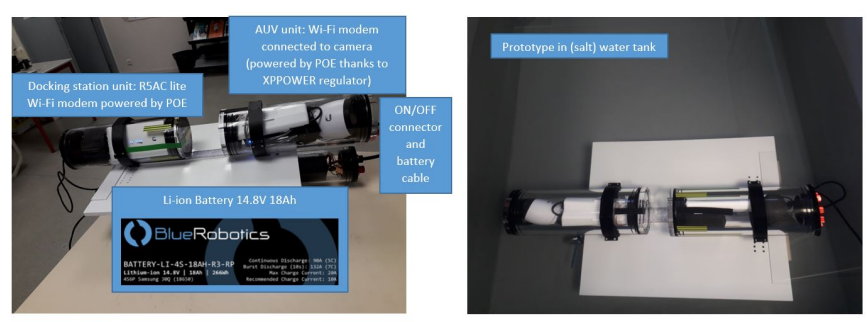

Fig. 1. airMAX RF contactless communication experimentation prototype.

\section{MI UNDERWATER COMMUNICATION}

As an emerging and promising alternative to traditional underwater communication techniques, MI communications have attracted significant attention in the past ten years [5], [8], [9]. Based on the Faraday's electromagnetic induction law, MI communication systems employ a couple of wired coils, where a modulated sinusoidal current at the transmitting coil induces an equivalent sinusoidal current at the receiving coil, that can be demodulated to retrieve the transmitted data.

\section{A. Studied ICPT system}

In this experimentation, we take as starting point the ICPT prototype developed in [1] providing wireless power transfer for AUV as illustrated in Fig. 2. The primary coupler is

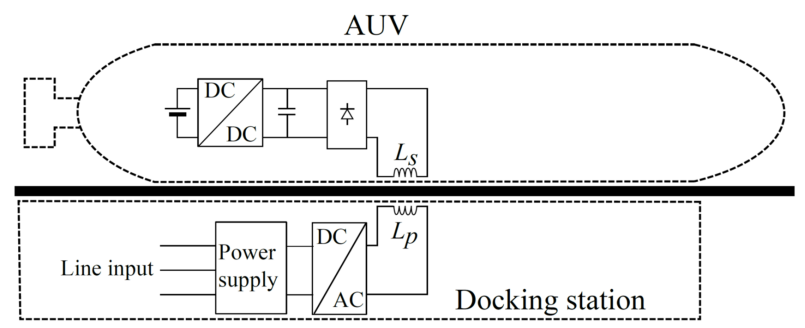

Fig. 2. Docking station for contactless underwater charging.

driven by a DC supplier with a constant current and an adjustable frequency inverter. The secondary coupler is linked to a (rectifier) with a variable resistance load. The pairs of couplers were made for a resonant $50 \mathrm{kHz}$ frequency.

\section{B. Achievable rate of MI communication channel}

The objective consists in using the ICPT system to also transfer data by magnetic coupling by using frequency bands beyond those used for power transfer. Typically MI communication systems operate at kilo Hertz frequencies over a transmission range of 10 to 100 meters [5]. In this paper, we consider contactless data transmissions with center frequency of the order of a few Mega Hertz. Through an impedance analyzer, we measured the amplitude and phase of the MI communication channel in the frequency band from 0.5 to 11 $\mathrm{MHz}$ as shown in Fig. 3. This MI communication channel of the ICPT prototype is first measured in air, fresh, and salt water. This sounded channel is interpolated at sampling 
frequency $f_{s}=B$, where $B$ denotes the bandwidth of the data transmission system, leading to the MI communication Channel Frequency Response (CFR) represented by fading coefficient $H_{n}$.

By assuming wideband transmission, the channel is demonstrated to be frequency selective, thus we assume a traditional Orthogonal Frequency Division Multiplex (OFDM) transmission system where data is modulated over $N_{c}$ orthogonal subcarriers spaced by $\Delta f \mathrm{~Hz}$ and spread over the whole bandwidth such as $B=N_{c} \Delta f$ [10]. In the case of a OFDM transmission, the channel capacity representing the maximum achievable rate for the system can be derived as [11]:

$$
C=\Delta f \sum_{n=0}^{N_{c}-1} \log _{2}\left(1+\frac{P_{n}\left|H_{n}\right|^{2}}{\sigma_{w n}^{2}}\right)
$$

where $P_{n}$ denotes the power assigned to subcarrier $n$ and $\sigma_{w n}^{2}$ the variance of the additive noise on subcarrier $n$ assumed Gaussian and zero mean. The power allocation policy which maximizes the capacity is specified by the waterfilling rule [12] :

$$
P_{n}= \begin{cases}\eta-\frac{\sigma_{w n}^{2}}{\left|H_{n}\right|^{2}} & \text { if }\left|H_{n}\right|^{2}>\frac{\sigma_{w n}^{2}}{\eta} \\ 0 & \text { else }\end{cases}
$$

with $\eta$ chosen such that $\sum_{n=0}^{N_{c}-1} P_{n}=P$ where $P$ denotes the total transmission power. Such allocation policy requires the knowledge of Channel State Information (CSI) at the transmitter side. The latest assumption appears realistic since the MI communication channel is known to be stable in time [5] and makes possible a transmission of CSI estimated at the receiver to the transmitter with a feedback loop.

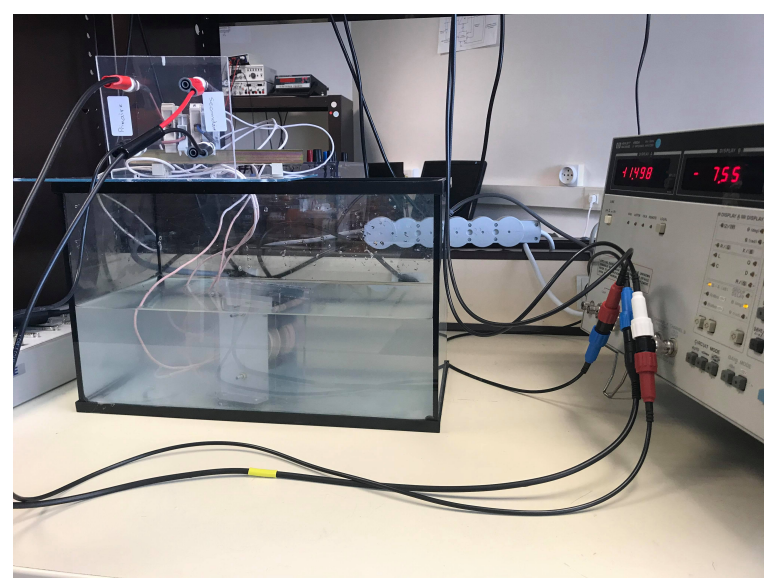

Fig. 3. MI communication channel measurement setup.

\section{EXPERIMENTAL RESULTS}

\section{A. Wi-Fi experiment results}

A range test for $27 \mathrm{dBm}$ power transmit level, which is the maximum level available, was first performed. The measured data rate at the receiver is shown Fig. 4 whereas the signal power strength at the receiver is shown Fig. 5.

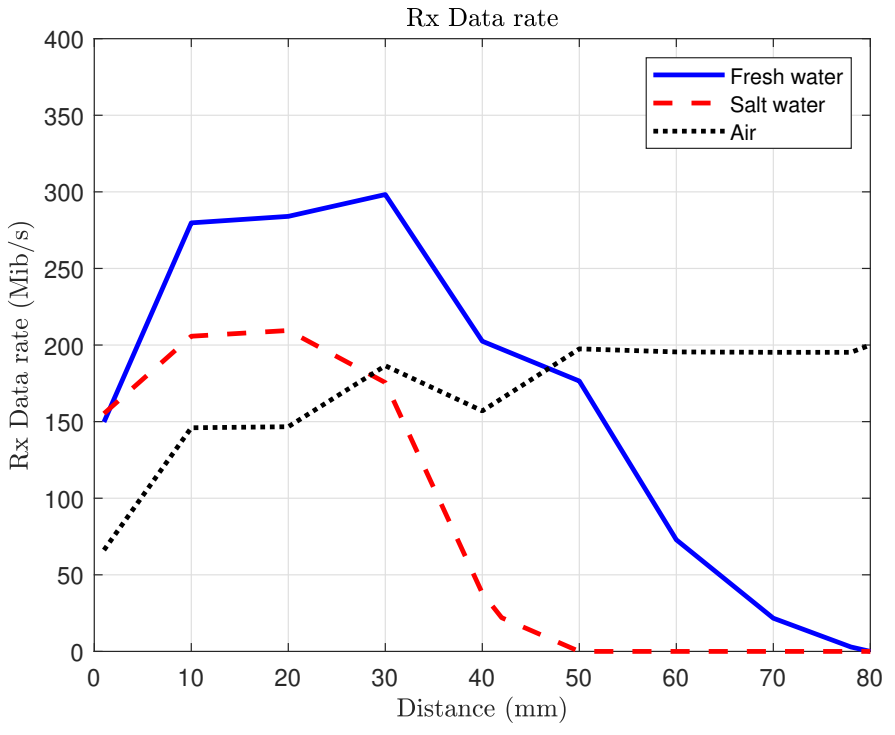

Fig. 4. Rx data Rate at $23 \mathrm{dBm}$ AUV transmit power.

In both air, fresh and salt, we observe a saturation data rate due the the very shirt distance between modems that can be explained by the fact that such modem are not designed for very short range communication. Whereas performance in air are quite stable as the range increases, data rates of underwater radio communication are dropping, faster in the case of salt water leading to a maximum transmission range in salt water corresponding to half of it in fresh water.

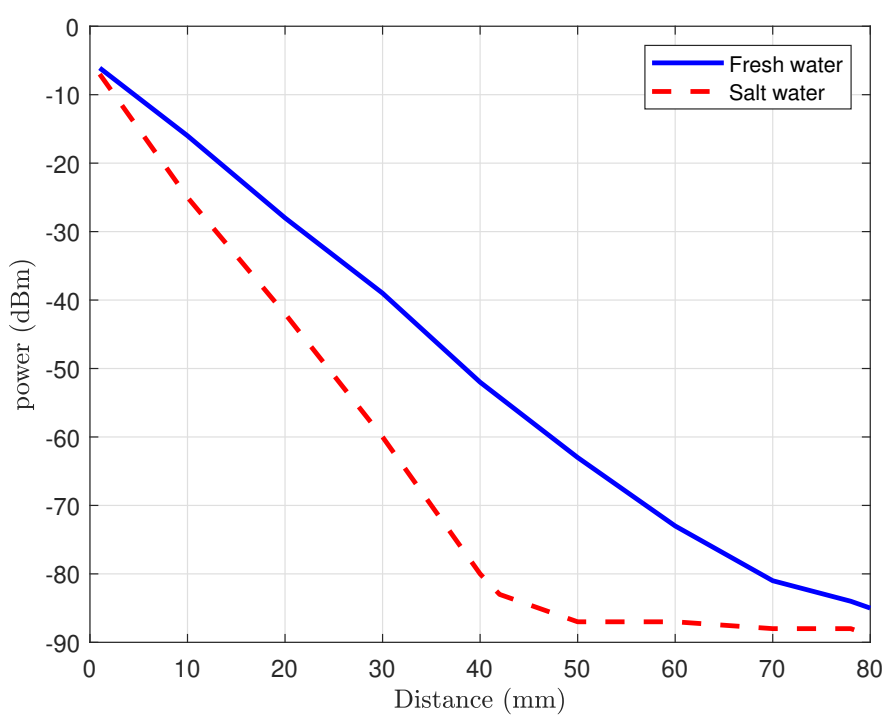

Fig. 5. Signal strength at Rx.

This dependency in range is illustrated in Fig. 5 which represents the signal strength at the receiver as a function of the distance. We can see the attenuation is quite linear with steering coefficients of $-997 \mathrm{~dB} / \mathrm{m}$ and $1875 \mathrm{~dB} / \mathrm{m}$ in fresh water and salt water respectively that confirm the theoretical radio wave attenuation in water presented in section II. 
Figs. 6 and 7 represent the influence of rotation and misalignment of the tubes on the data rate, respectively. We can see that except for one configuration, the impact of rotation is not significant. For one position, there is a $6 \mathrm{~dB}$ drop of the data rate in salt water and $3 \mathrm{~dB}$ drop data rate in fresh water. We can see that the impact of a small misalignment is not significant.

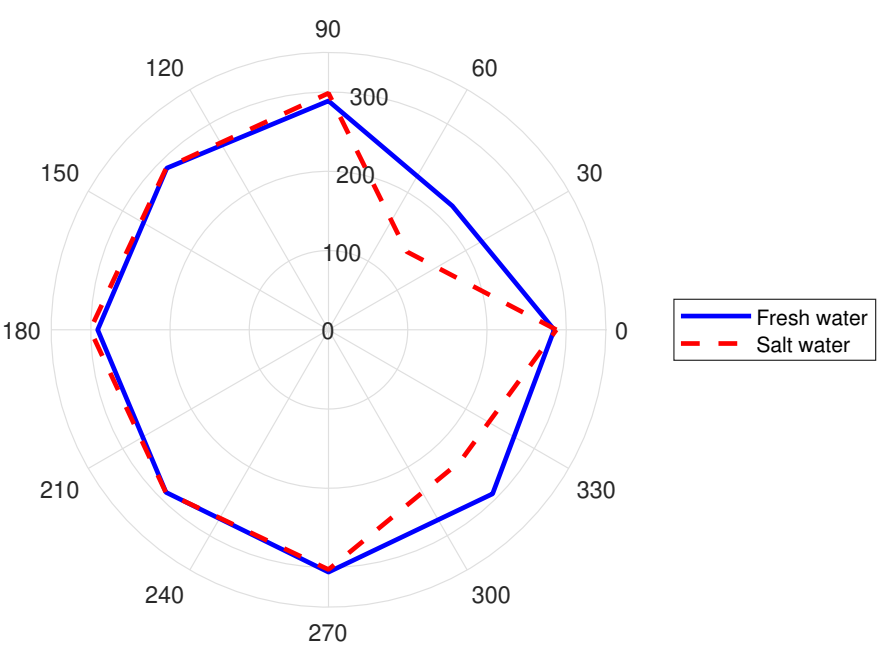

Fig. 6. Influence of rotation.

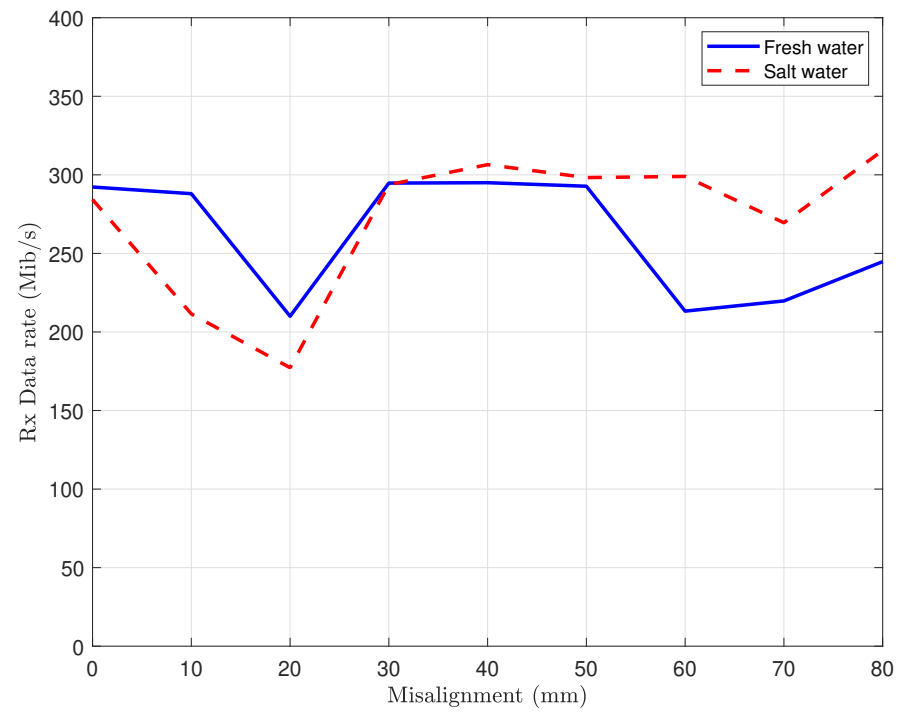

Fig. 7. Influence of misalignment.

\section{B. Induction simulation results}

The MI communication CFR is sounded by using the measurement set up described in Section III and plotted in Fig. 8 for a $4 \mathrm{~mm}$ spacing between the primary and secondary magnetic couplers. Both transmission directions (i.e. primary to secondary and secondary to primary) are sounded in order to evaluate the scenario where the docking station upload data to the AUV (mission plan for example) and reversely the AUV data (video, SONAR images) are downloaded to the docking station. In the air, the CFR appears with a resonant frequency at $1.4 \mathrm{MHz}$ in case of primary-to-secondary and $1.2 \mathrm{MHz}$ in the reverse direction, whereas in fresh or salt water the resonant frequency appears to be between 7.2 and $8.5 \mathrm{MHz}$ with a larger peak than in air.

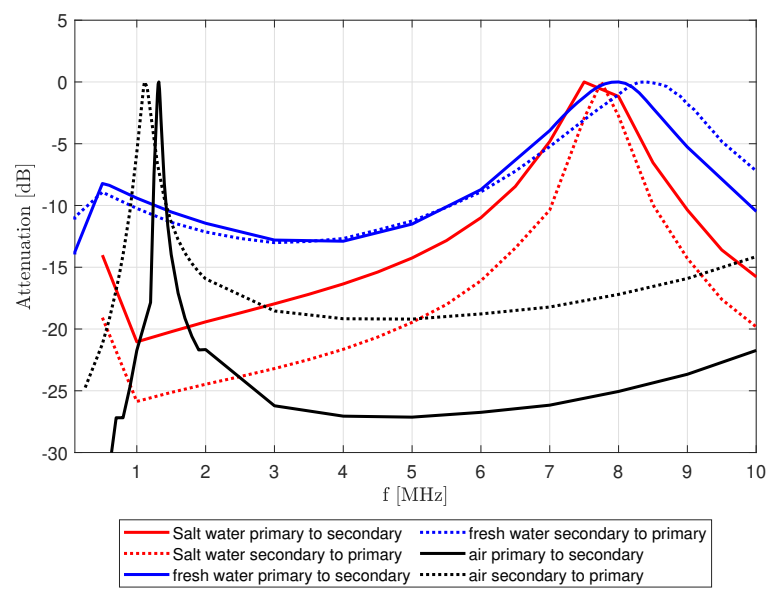

Fig. 8. MI communication channel measurements.

Fig. 9 provides an example of MI communication channel capacity results as function of the Signal-to-Noise Ratio (SNR) by feeding previously sounded CFR into the expression (1) and by assuming a center frequency of $5 \mathrm{MHz}$ and a bandwidth of $9 \mathrm{MHz}$. As benchmark we add so-called Gaussian capacity $C_{\mathrm{AWGN}}=B \log _{2}(1+\mathrm{SNR})$ representing an upper bound on frequency selective channel capacity. Due to its lowest frequency selectivity, best performance are achieved for MI transmission in fresh water whereas in sea water the maximum achievable spectral efficiency is estimated at $5.1 \mathrm{bps} / \mathrm{Hz}$ in case of primary-to-secondary transmission and $4.5 \mathrm{bps} / \mathrm{Hz}$ in the reverse direction leading to potential date rate $46 \mathrm{Mbit} / \mathrm{s}$ and $51 \mathrm{Mbit} / \mathrm{s}$ respectively.

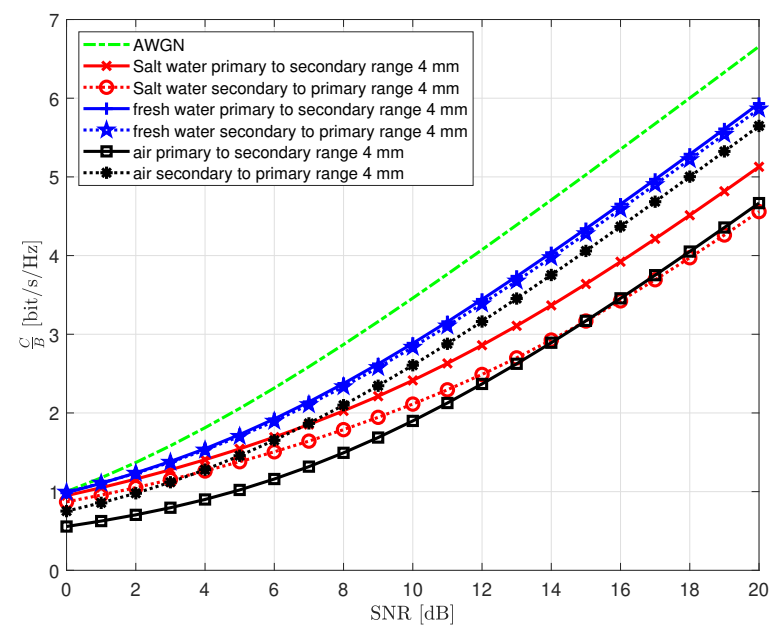

Fig. 9. MI communication channel capacity for center frequency $f_{0}=5$ $\mathrm{MHz}$ and bandwidth $B=9 \mathrm{MHz}$. 


\section{CONCLUSIONS AND DISCUSSION}

The objectives of this paper was to investigate two contactless radio-based data transfer systems in the framework of AUV battery recharge with an underwater docking station by using an ICPT system. A 802.11ac Wi-Fi system is experimentally demonstrated to achieve up to $300 \mathrm{Mbps}$ payload in salt water with low sensitivity to misalignment and rotation. The second approach aims to reuse the ICPT system to transfer data in addition to power by magnetic coupling. By sounding experimentally the MI communication channel, capacity analysis suggests an achievable rate in the order of 50 Mbits, a payload rate 6 times lower than in Wi-Fi approach but using the same transducer for data and power transfer thus requiring less integration effort in the AUV than in a Wi-Fi based transfer system. Future works will address interference issues between ICPT and MI communication systems as well the choice of the optimum coupler for both data and power transmission.

\section{REFERENCES}

[1] Y. Amirat, G. Feld, and Y. Auffret, "Performance Analysis of an Inductive Contactless Power Transfer System," in IECON 2020 The 46th Annual Conference of the IEEE Industrial Electronics Society, Oct. 2020, pp. 3918-3923.

[2] M. Fewell, A. von Trojan, and M. Steed, "Empirical Range-DataRate Relationships for Undersea Wireless Communications Systems," Defense Science and Techology Group, Australian Government, General Document DSTG-GD-1101, Oct. 2020.

[3] M. Stojanovic and P.-P. J. Beaujean, "Acoustic Communication," in Springer Handbook of Ocean Engineering, M. R. Dhanak and N. I. Xiros, Eds. Springer International Publishing, 2016, pp. 359-386.

[4] S. Sendra, J. Lloret, J. M. Jimenez, and J. J. P. C. Rodrigues, "Underwater Communications for Video Surveillance Systems at $2.4 \mathrm{GHz}$," Sensors, vol. 16, no. 10, p. 1769, Oct. 2016.

[5] Y. Li, S. Wang, C. Jin, Y. Zhang, and T. Jiang, "A Survey of Underwater Magnetic Induction Communications: Fundamental Issues, Recent Advances, and Challenges," IEEE Communications Surveys Tutorials, vol. 21, no. 3, pp. 2466-2487, thirdquarter 2019.

[6] S. Sendra, J. Lloret, J. M. Jimenez, and L. Parra, "Underwater Acoustic Modems," IEEE Sensors Journal, vol. 16, no. 11, pp. 4063-4071, Jun. 2016.

[7] F. Teixeira, P. Freitas, L. Pessoa, R. Campos, and M. Ricardo, "Evaluation of IEEE 802.11 Underwater Networks Operating at $700 \mathrm{MHz}, 2.4$ $\mathrm{GHz}$ and $5 \mathrm{GHz}$," in Proceedings of the International Conference on Underwater Networks \& Systems, ser. WUWNET '14. New York, NY, USA: ACM, 2014, pp. 11:1-11:5.

[8] Y.-g. Huang, C. Fang, and X.-f. Li, "Contactless power and data transmission for underwater sensor nodes," EURASIP Journal on Wireless Communications and Networking, vol. 2013, no. 1, p. 81, Mar. 2013.

[9] M. Hott and P. A. Hoeher, "Underwater Communication Employing High-Sensitive Magnetic Field Detectors," IEEE Access, vol. 8, pp. 177 385-177 394, 2020.

[10] J. G. Proakis and M. Salehi, Digital Communications 5ed, 5th ed. Boston, Mass.: McGraw-Hill, 2008.

[11] D. Tse and P. Viswanath, Fundamentals of Wireless Communication. Cambridge University Press, 2005.

[12] Y. M. Aval, S. K. Wilson, and M. Stojanovic, "On the Achievable Rate of a Class of Acoustic Channels and Practical Power Allocation Strategies for OFDM Systems," IEEE Journal of Oceanic Engineering, vol. 40, no. 4, pp. 785-795, Oct. 2015. 